\title{
Research on the Music Style of Clarinet Performance
}

\author{
Peilong He \\ Xi’an Conservatory of Music, Xi'an, Shaanxi, 710061
}

Keywords: Clarinet Performance, Music Style, Development Trend

\begin{abstract}
In this paper, the development history of the clarinet and the structure of the clarinet are summarized. Then, from the aspects of the performance of the clarinet, the teaching method is explained by the type, tone, pronunciation, spit, hand and transit of the clarinet. In the teaching of students prone to the situation to analyze and solve, and playing on the method of teaching some of the views; on the psychological also carried out a brief overview, combined with the above, on the teaching methods of teaching, conducted some discussion ; Playing not only have skills, but also music, for the performance of the works and playing style, through the clarinet each period of representative composers and works for a brief analysis of the style of the song and there are some music on the Playing, summed up from the classical period to the century, the characteristics of various periods of music and style, so as to how the players to form their own style of play to discuss.
\end{abstract}

\section{Introduction}

After the medieval music, the Renaissance and Baroque period, the beginning of the century, the clarinet was born. The birth of a clarinet is usually considered to be invented by John Christopher, an outstanding woodwind instrumentist in Nuremberg, even though the invention of the clarinet is not due to him, but he is still an excellent producer. In the early part of the clarinet, there was a period called Shalumu tube that had been with him for some time, which made the history of the period of the clarinet less clear. But it is certain that the appearance of the 'overtone key' is the sign of the single-yellow tube, which allows the sound of the single-han tube and the sand lu-yu tube to exist simultaneously on a clarinet.For who invented the clarinet At the beginning, the clarinet's flute head was seen as the top of the instrument, and as the performance and the development of the instrument, people gradually saw the flute head to the sound The importance of the production of the whip head more sophisticated in the record of Beckham has mentioned: "In that era of players, about people use two ways (praise on this or under) In 1831, the Paris Conservatory of Music officially changed the position of the reeds on the lower lips, which is the basic shape of the flute we now see, and the early flute has a tapered profile and a rather narrow inner groove, The matching reed is not only narrow and very short, but later, as the flute head gradually widened, the flute head facing the tip of the tip shape is also more and more obvious, and in the sealing this was a certain degree of curvature. Head of the production Materials, producers from the beginning of the wood (such as boxwood or fruit) to gradually use some anti-moisture materials, to the middle of the 20th century, with hard glue to do the head, to now, the plastic has become the main The production of the material (also made of glass) is also widely used in beginners, and the reed is also improved with the development of the flute head. Wide (and flute head) and thick, and not to the top of the thinner; to the 19th century, the British appeared in the shape is very close to the modern we used reeds, and to the modern, we used reed condition, German style, the length of their thickness, width and width are different, to use with its shape, profile, bore, width and width match the flute head.It can be seen, modern clarinet flute head and whistle the production of fine.

\section{Single Praise of the Playing Method}

For a beginner, there is a correct guide and method, then both in the play or in the future play has 
important significance. The legs, chest, neck, knee to relax this can naturally breathe and can easily find the correct pronunciation feeling, and playing the two feet naturally stand, probably shoulder width, chest up, upright, Good posture. The two arms relax naturally droop, and then lift the arm, left hand in the right hand in the next, then, pick up the black tube, right thumb into the next section of the instrument under the care, hold the black tube, the rest of the natural ride in front Of the sound hole, arms to maintain a natural relaxation; as just beginning to learn, you can first use the left hand to hold the single tube of the two sections to ease the right hand thumb black tube power. After taking the instrument, the instrument and the body into a 40-45 degree angle. When the head into the mouth, the upper teeth (two front teeth) into the slope on the front of the head above the smooth, about one-third (about one centimeter, the upper lip naturally carrying the head, (Not too much, not too little, according to the individual lips of the thick, comfortable, natural, moderate can), naturally closed (similar to the "double bag" type, but not "double bag" , Face relaxed, mouth slightly smiling; mouth-shaped, with the tongue gently close to the reed (with the tip of the tongue close to the top of the whistle about half a centimeter, in the breath and tongue out of the whistle of the moment, issued The lower lip is not too much, the upper lip and the lower lip in appearance, the lower lip is less than the upper lip in the flute head position, so easier to make a good sound. Because beginners on the tongue and pronunciation is not very Cooked, the pronunciation can be no first tongue, the first sound of the instrument. Then we say the mouth of the internal, to issue a nice voice, not only have the correct mouth, but also the mouth, breath, tongue and even fingers With the mouth Part is very important, it is implicated to change the sound.

\section{Clarinet Music Historical Style Evolution}

Classical music, it is in the ideological freedom of cultural requirements and secular feelings of human performance under the dual influence, took the Renaissance, Baroque music culture, to the solemn and not stiff, free and without losing, unrestrained, Elegant and not wild aesthetic expression of the direction of development, will be no title (or pan title) of the universal beauty and common sense of the common sense of pleasure in the unity of formal beauty in the universal value experience, the program requires a strict order Into the aesthetic sense of the emotional performance of harmony in the aesthetic sense of unity in the general sense of elegance. This universal value experience and general and elegant feeling, in the Mozart "A major clarinet concerto" made a concrete presentation, and become in the clarinet of this instrument on the beautiful music of the classic.

Romantic art style features, embodied in the music, that is manifested as: on the one hand, the creation of new forms of expression, such as the four movement of the symphony of the strict norms of the program by two movements, five movements of the symphony program breakthrough, Single concert of the concerto also broke through the three fixed movement of the specification requirements, romantic songs, fantasy trees mushrooming constantly emerging, symphonic poems is the music genre expression of the new work. On the other hand, the romantic music from the general sense of rational thinking to the specific bearing of the emotional performance, from the spirit of the request to the emotional change, from the cultural investigation to the self-catharsis of this cultural conception and aesthetic thrust of the direction of the transfer, The full expression of the artistic personality and the full expansion of the emotional self-expression leapt to the new starting point of the historical logic of music thinking. It is not only the logical inheritance of classical music, but also the aesthetic new performance of the music culture.

In the second half of the nineteenth century, the romantic poems that emerged from France, the uncertain semantics, the hazy consciousness, the linguistic way of perturbation and the perishable semantic expression, deeply influenced the aesthetic pursuit of Debussy's music creation. We often say that the two factors of Impressionist painting and symbolism have influenced the formation of Debussy's impressionist musical style, and that the influence of Impressionist painting on Debussy is greater than that of symbolism. In fact, for Debussy, the influence of symbolism on his poems is much greater than the influence of Impressionist painting on his creation of Impressionist music.

Romantic music, by the European overall cultural background and artistic style requirements of 
the changes in the impact of its musical style and classical music aesthetic compared to both in the form and content, have a different track of the great differences between the car. As a principle, romantic art and classical art of cultural differences and aesthetic ideals, mainly reflected in the personality of the awakening and emotional publicity in the two major directions. Classical art style requirements, reflecting the universal beauty and universal values of the highest principles of artistic principles. Common beauty and universal value, together cast the classical art of the macro point, but the romantic art of the greatest feature of the tendency is the common beauty and universal value of the negative and beyond, the common beauty gave way to the beauty of the beauty of the strong confession, The universal value also gave way to the personality of the self-elucidated, personalized publicity and emotional surge, wake up sleeping in the hearts of people's self-consciousness, sensibility beyond the rational, individual beyond the general, emotional beyond reason, freedom beyond the law, Personality feelings of publicity and emotional consciousness of the catharsis, the impact of classical art norms of the lofty ideals, but also people's artistic style requirements into a new world of art show.

Impressionist music, after experiencing the classical music aesthetic synaesthesia, universal values and romantic music personality publicity, emotional marking after the historical period, the other to see, in the misty imagination of the moment on the image, deliberately depict the light color to the moment Feelings, in the following two points to make transcendental conception change, one is to spin the law to describe the characteristics of the second is the use of harmony on the color. In the spin method, Debussy changed from Baroque music, classical music since the narrative characteristics, instead of the description of the music language to start his musical creativity. In harmony, the function of harmony in its creative case is the end, replaced by color and harmony to render it the unique mood of the music mood. In the clarinet playing, from the following aspects to be carved more appropriate: 1 . Impressionist style of music, determines its music expression should not be large span of emotional contrast and great intensity of emotional performance, changes in the subtle, statements of subtle, confession of the introverted, lyrical possession of the front, is to grasp the basic requirements of Impressionist music style, Grasp this point, put the impression of the basic style of Impressionist music style, but also the characteristics of Impressionist music style of the eye-catching. In the intensity of performance, to be appropriate to have degrees, subtle see the big chapter, quiet place to see the effect, the romantic music that kind of cross knife vertical ax, here is not see its meritorious service, but not the intensity of tiling, to promote the balance, but a description of the Jiedu, moderate infection, euphemism and the constraints of the Tibetan front, grasp the appropriate measurement, Impressionist music basic style of performance in the palm Among the shares. 3. The direction of transport. Impressionist music style melody, decided that its spin method is basically separated from the natural sound level formed in the orderly harmonic and the decomposition of the chord of the spin direction, depicting the characteristics of the point of view, so that its spin to point strokes Characteristics, therefore, have to change the normal behavior of our normal behavior, a variety of non-natural sound level and interval of the jump is moving the meaning of the impression that the essence of the impression that the impression, grasp this point, Impressionist language And smooth and coherent music show can be reflected.

The above three aspects, is to embody the Impressionist music style must be carefully considered the support point, but also the interpretation of Impressionist music style must have the guiding principles, omitting meaning once understood, the natural embodiment of the style will be a matter of course.

\section{Conclusion}

With the development of the Renaissance humanistic self-awakening and secularization of music, the music is toward Baroque, the development of classical music, aesthetic sensibility and universal value. It represents the aesthetic ideal of classical music, and the personality is reflected. To send the typical characteristics of the music style, instant feelings, misty fantasy, but also Impressionist music is different from the classical, romantic style of music performance. Impressionist music depicts the historical features of the classical, romantic music narrative features, opened the history 
of the development of modern music in Europe, and influenced the 20th century, all kinds of modern music development history.

\section{References}

[1] Zhang Yuying. On the piano music "Pinghu moon" artistic characteristics [J]. Northern Literature. 2017 (24)

[2] Zha Mu Su. Ma Touqin art and playing style research [J]. Northern music. 2017 (15)

[3] Wang Anlin. Giovanni Bortecini bass violin creation and playing style analysis [J]. Tomorrow fashion. 2017 (19)

[4] Gao Yu. On the teaching of students in the style of playing style [J]. Music time and space. 2016 (02)

[5] Li Yuxuan. Chinese piano works playing style interpretation [J]. Northern music. 2015 (12)

[6] Wang Deyi. Mozart piano music playing style [J]. Big stage. 2014 (06) 